\title{
Chemical Characterization of Denture Base Resin with a Novel Cycloaliphatic Monomer
}

\author{
Ranganthan Ajay ${ }^{1}$, Karthigeyan Suma², Sampathkumar JayaKrishnaKumar ${ }^{3}$, Gunaseelaraj Rajkumar ${ }^{4}$, \\ Sengottaiyan Arul Kumar ${ }^{5}$, Radhakrishnan Geethakumari ${ }^{6}$
}

\begin{abstract}
Aim: The aim of this study is to identify and characterize newly formed copolymers by modifying methyl methacrylate (MMA) monomer by substituting cycloaliphatic monomer using Fourier transform infra-red (FTIR) spectroscopy.

Materials and methods: Heat-cure polymethyl methacrylate (HC-PMMA) experimental specimens were made by dissolving tricyclodecane dimethanol diacrylate (TCDDMDA) at $10 \%$ and $20 \%$ concentrations in commercially available MMA monomer. Specimens made without TCDDMDA served as the control. The specimen was then scrapped and mixed with dried potassium bromide (KBr) to form pellet. Each pellet was placed in a FTIR spectrometer and 10 scans were recorded with a spectral resolution of $4 \mathrm{~cm}^{-1}$. A mean of scans was automatically processed and deduced by the system software and a final transmittance spectral graph was obtained for one specimen.

Results: Three significant spectral differences exist between control and experimental groups. The first difference was the disappearance of weak peak at $1637.34 \mathrm{~cm}^{-1}$ (alkenyl $\mathrm{C}=\mathrm{C}$ stretch) in both experimental groups. The second difference was the appearance of new moderate broad peaks at $1482.25 \mathrm{~cm}^{-1}$ and $1449.56 \mathrm{~cm}^{-1}$ in both experimental groups which are attributed to the ring $-\mathrm{CH}_{2}$ asymmetric bending $(\mathrm{C}-\mathrm{H}$ deformation) vibrations. The third difference was the appearance of another new weak peak at $1386.57 \mathrm{~cm}^{-1}$ in both experimental groups. This new peak confirms the formation of a new structure of copolymer.

Conclusion: TCDDMDA copolymerizes with MMA, thereby decreasing the uncured residual monomer in the polymerized specimens.

Clinical significance: Copolymerization of TCDDMDA with MMA would lead to the development of new monomeric composition for the fabrication of dentures possessing better mechanical properties and biocompatibility.

Keywords: Copolymer, Cycloaliphatic compound, Monomer, Modified monomer.

The Journal of Contemporary Dental Practice (2019): 10.5005/jp-journals-10024-2634
\end{abstract}

\section{INTRODUCTION}

Poly(methyl methacrylate) (PMMA) is the most commonly used the denture base material for the past eight decades. This has remained the denture base material of choice although various polymers have been developed and introduced in dentistry to overcome the deficiencies of PMMA. This can be attributed to the fact that though the properties of PMMA are not ideal in every aspect, it is the combination of various rather than one single desirable property that accounts for their popularity and universal use. ${ }^{1}$

Various investigations have shown the limitation of HC-PMMA resin with respect to dimensional accuracy and strength, particularly under impact and fatigue conditions. ${ }^{2}$ Various investigators have compared the dimensional changes among different denture base materials, ${ }^{3,4}$ palatal vault configurations, ${ }^{5}$ methods of packing, ${ }^{6-8}$ modes of polymerization, ${ }^{2,9,10}$ and curing cycles. ${ }^{11}$ The researches of these investigators showed that there were definite dimensional changes of varying magnitude occurring in HC-PMMA. Nevertheless, cytotoxic effects of these resins were also studied by numerous researchers.

Several modifications have been experimented in the denture base acrylic resins to improve the physico-mechanical properties and biocompatibility. These modifications can be broadly classified into polymer and monomer modifications. The polymer modifications are done by either chemically modifying polymers or adding inorganic compounds and organic fibers. The advances in polymer technology have produced novel polymers with improved impact strength and fatigue resistance. However, the reinforcement fillers like rubber ${ }^{12,13}$ and fibers ${ }^{14}$ to HC-PMMA
1,6 Department of Prosthodontics and Crown and Bridge, Vivekanandha Dental College for Women, Namakkal, Tamil Nadu, India

${ }^{2}$ Department of Prosthodontics and Crown and Bridge, Rajah Muthaiah Dental College and Hospital, Annamalai University, Chidambaram, Tamil Nadu, India

${ }^{3}$ Department of Prosthodontics and Crown and Bridge, Ragas Dental College and Hospital, Chennai, Tamil Nadu, India

${ }^{4}$ Department of Prosthodontics and Crown and Bridge, Vinayaka Missions Sankarachariyar Dental College and Hospital, Salem, Tamil Nadu, India

${ }^{5}$ Department of Prosthodontics and Crown and Bridge, Sri Venkateshwara Dental College and Hospital, Chennai, Tamil Nadu, India

Corresponding Author: Ranganthan Ajay, Department of Prosthodontics and Crown and Bridge, Vivekanandha Dental College for Women, Namakkal, Tamil Nadu, India, Phone: +91 8754120490, e-mail: jrangclassiq@gmail.com

How to cite this article: Ajay R, Suma K, et al. Chemical Characterization of Denture Base Resin with a Novel Cycloaliphatic Monomer. J Contemp Dent Pract 2019;20(8):940-946.

Source of support: Nil

Conflict of interest: None

polymer not only improve mechanical properties but may also affect dimensional accuracy and stability. In the recent years, chemically modified monomers with good cytocompatibility,

() The Author(s). 2019 Open Access This article is distributed under the terms of the Creative Commons Attribution 4.0 International License (https://creativecommons. org/licenses/by-nc/4.0/), which permits unrestricted use, distribution, and non-commercial reproduction in any medium, provided you give appropriate credit to the original author(s) and the source, provide a link to the Creative Commons license, and indicate if changes were made. The Creative Commons Public Domain Dedication waiver (http://creativecommons.org/publicdomain/zero/1.0/) applies to the data made available in this article, unless otherwise stated. 
high dimensional accuracy, and improved strength have been developed. Unlike polymeric modifications, there are only a few researches available pertaining to monomer modifications. Monomers such as fluoromonomers, phosphate monomers, methacrylic acid monomer, itaconate monomers, nitro-monomers, and other nonspecific monomers have been substituted with MMA and the physico-mechanical properties of $\mathrm{HC}$ denture base resins were studied. ${ }^{15}$ Monomer modifications were done by a volume replacement of MMA at various concentrations. Except methacrylic acid monomer, copolymerization or chemical characterization of all the above monomers has not been documented in the dental literature. The formation of new copolymers has a positive correlation with respect to material's properties. ${ }^{15}$

TCDDMDA is a novel dual reactive, cyclo-aliphatic, colorless, and odorless acrylic monomer, capable of further reaction by incorporating new functionality, grafting new polymeric chains, attaching drugs or biomolecules. This monomer has easily polymerizable carbon-carbon $(C=C)$ double bonds. Yet, it also contains a secondary reactive group that can be manipulated in a multitude of ways. The tricyclodecane three-ring central group prevents monomers from aligning, and, thus, offsets polymerization shrinkage. ${ }^{16}$ The steric hindrance effect of this cyclic ring structure slows the polymerization rate and facilitates adjacent polymeric chains to slip among themselves for stress relaxation before reaching the point of solidification.

Tricyclodecane-based or cycloaliphatic monomer has been used in the field of dentistry as a bulky, space-filling monomer in resin matrices of resin-veneered metal restorations and proven to have superior physical properties to those of conventional resins. ${ }^{16}$ Tricyclodecane monomers also possess excellent hydrolytic stability, good abrasion resistance, and toughness and, hence, used in various industrial applications such as coatings on automotive, metal, glass, and plastic.

However, the chemical characterization of TCDDMDA substituted in MMA and its effect on the properties of HC-PMMA has not been documented so far in the dental literature. Hence, the study aims to identify and characterize any newly formed copolymeric structures by modifying MMA by substituting TCDDMDA at concentrations of $10 \%$ and $20 \%$ using FTIR spectroscopy.

\section{Materials and Methods}

This study was conducted at the Department of Chemistry (Organic), Annamalai University, Chidambaram, Tamil Nadu. Heat-cure acrylic resin (DPI, Mumbai Burmah Trading Corp Ltd, Mumbai, India) and TCDDMDA monomer (Sigma-Aldrich Co. St. Louis, USA; Product no. 496669) were used in this study. Control group: C0 comprises 10 specimens made without substituting TCDDMDA in MMA (100\% MMA). Experimental groups: C10 and C20 have 10 specimens each prepared by substituting TCDDMDA in MMA at $10 \%$ and $20 \%(\mathrm{v} / \mathrm{v})$ concentration, respectively. The substituted monomers were stored in three separate identical dark glass bottles and labeled with appropriate concentrations. Blinding the author was accomplished by concealing the labels with opaque stickers and randomly numbering them from one to three (Bottles 1, 2, and 3) to avoid expectation bias. For each group, 10 test specimens were prepared with standardized disc shape (25 mm diameter: Fig. 1A; $3 \mathrm{~mm}$ thickness: Fig. $1 \mathrm{~B})^{17}$ to evaluate copolymerization of TCDDMDA with MMA by using FTIR. Steel dies were impressed in the putty impression (Photosil ${ }^{\text {TM }}$ Soft putty; DPI; The Mumbai Burmah Trading Corp. Ltd, Mumbai, India) in the dental flask to obtain mold space. According to manufacturer's instruction, the polymer and the monomer were proportionated at 3:1 ratio in a porcelain jar and kept closed until the mix reached the dough stage. The dough forming time was 8 minutes for the control group and 15 minutes for experimental groups. The mold spaces were then packed with the dough at a packing pressure of 3,500 psi in mechanical press (Sirio Dental Srl, Meldola FC, Italy) for 10 minutes and then heat cured at $74^{\circ} \mathrm{C}$ for 9 hours in an acrylizer (Unident Instruments India Pvt. Ltd). After curing, the flasks were bench cooled to room temperature. Conventional trimmers and graded emery sheets were used for finishing and polishing. ${ }^{18}$ All the polymeric specimens were prepared by single investigator. The specimens were then stored in a container with distilled water for 48 hours with the same random number found on the monomer bottle. Hence, the monomer's concentration in the polymerized specimen was blinded. Then, the specimens were removed and dried in air for 24 hours to remove the water from the specimens.

The transmittance spectra were obtained in a FTIR spectrometer (ThermoScientific ${ }^{\mathrm{TM}}$ Nicolet $^{\mathrm{TM}}$ iS $^{\mathrm{TM}} 5$; iD1 Transmission, Waltham, MA; USA) (Fig. 2) using a spectral resolution of $4 \mathrm{~cm}^{-1}$ with a wavelength precision of $0.01 \mathrm{~cm}^{-1}$ at $2000 \mathrm{~cm}^{-1}$. The dried specimens were scraped by using a sharp sterile wax $\mathrm{knife}^{18}$ to form $2 \mathrm{mg}$ of small pieces and mixed with $100 \mathrm{mg}$ of dry potassium bromide $(\mathrm{KBr})$ powder. ${ }^{19}$ The mixture was pressed into a disk of $0.5-\mathrm{mm}$ thickness using special mold and hydraulic pellet press (Kimaya Engineers; Thane, Maharashtra, India) (Fig. 3). The operator was blinded from the composition of the specimens while preparing the disk pellets and interpreting the transmittance graph on the computer. The disk pellets were vertically placed on a specimen holder compartment (Fig. 4). Infra-red beam was transmitted through the surface of the sample. The beam passed to deuterated tri-glyceride sulfate (dTGS) detector for a final quantitative measurement. The detector measured the special interferogram signal and fed to the computer in digitized (OMNIC ${ }^{\text {TM }}$ software) form for interpretation. ${ }^{17}$ All transmitted spectra were obtained from 10 scans and averaged at $4 \mathrm{~cm}^{-1}$ spectral resolution. ${ }^{20}$

\section{Results}

For both control and experimental groups, infrared spectra analysis displayed several peaks of PMMA specimens. The transmission spectra were recorded in the range of $4000-600 \mathrm{~cm}^{-1}$ and serve as a direct means for the diagnosis of the function group of the given specimen. The FTIR spectrum of the control group: CO specimens are shown in Figure 5.

The spectrum depicts a weak to moderate, a peak at 3436.38 $\mathrm{cm}^{-1}$, which is attributed to the presence of $-\mathrm{CH}_{3}$ stretching vibrations. Peaks at $2995.60 \mathrm{~cm}^{-1}$ and $2951.53 \mathrm{~cm}^{-1}$ were owing to $\mathrm{O}-\mathrm{H}$ and methylene $\mathrm{C}-\mathrm{H}$ stretching vibrations. A strong peak at $1731.79 \mathrm{~cm}^{-1}$ is due to ester carbonyl $\mathrm{C}=\mathrm{O}$ stretching vibrations. The appearance of a weak peak at $1637.34 \mathrm{~cm}^{-1}$ is attributed to alkenyl $\mathrm{C}=\mathrm{C}$ stretch. Peaks at $1271.44 \mathrm{~cm}^{-1}$ and $1147.82 \mathrm{~cm}^{-1}$ were due to the presence of $\mathrm{C}-\mathrm{O}$ ester band and $\mathrm{C}-\mathrm{O}-\mathrm{C}$ asymmetric stretch vibrations, respectively. Peaks at $989.74 \mathrm{~cm}^{-1}, 963.76 \mathrm{~cm}^{-1}$, and $841.50 \mathrm{~cm}^{-1}$ were attributed to the $\mathrm{C}-\mathrm{H}$ out of plane bend. The peak at $750.92 \mathrm{~cm}^{-1}$ is due to cis-C-H out of plane bend.

Figures 6 and 7 show the FTIR spectra of experimental groups. There are three significant spectral differences between control and experimental groups. First is the disappearance of weak peak at $1637.34 \mathrm{~cm}^{-1}$ (alkenyl $\mathrm{C}=\mathrm{C}$ stretch) in both experimental groups. Second, the appearance of new moderate broad peak 

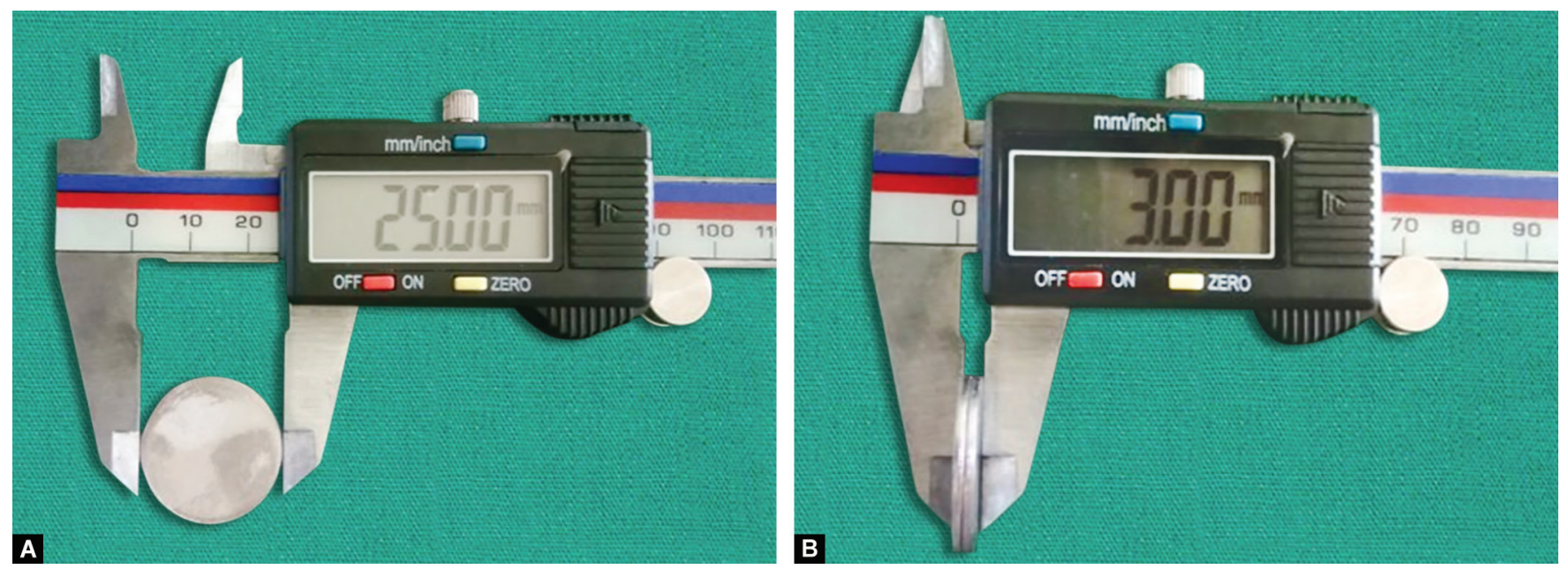

Figs 1 A and B: (A) Steel die diameter; (B) Steel die thickness

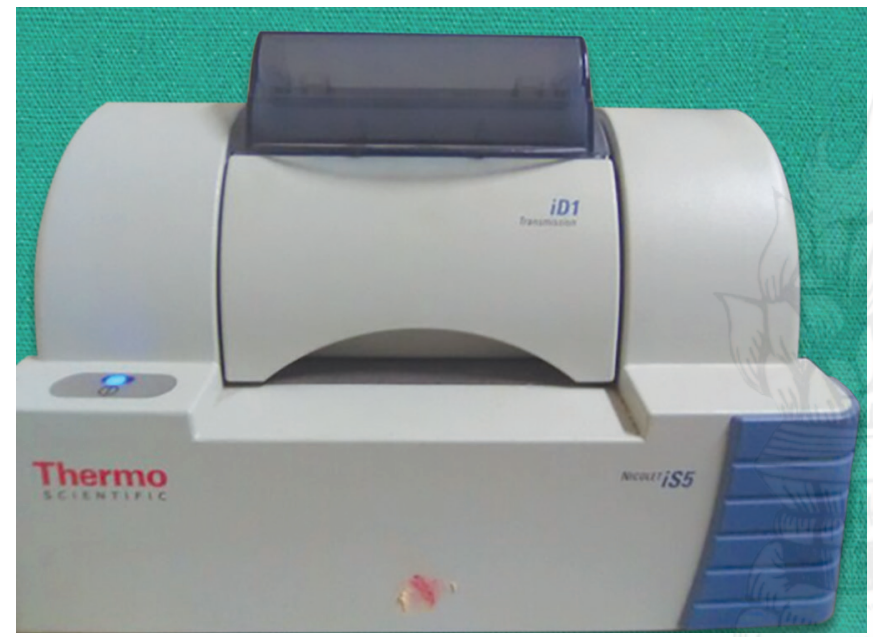

Fig. 2: FTIR spectrometer

between $1500 \mathrm{~cm}^{-1}$ and $1400 \mathrm{~cm}^{-1}$ (exclusively at $1482.25 \mathrm{~cm}^{-1}$ and $\left.1449.56 \mathrm{~cm}^{-1}\right)$ in both experimental groups which are attributed to the ring $-\mathrm{CH}_{2}$ asymmetric bending ( $\mathrm{C}-\mathrm{H}$ deformation) vibrations. The third difference is the appearance of another new weak peak at $1386.57 \mathrm{~cm}^{-1}$ in both experimental groups. This new peak confirms the formation of a new structure of copolymer. The remaining stretching vibrations were similar to the control group C0. Figure 8 highlights the spectral differences of the three groups. There were no significant spectral differences between the experimental groups. Table 1 describes the chemical characterizations of the groups. There is no significant difference between the transmitted spectra of experimental groups.

\section{Discussion}

Three principal spectral differences appeared among the groups. First is the disappearance of the peak at $1637.34 \mathrm{~cm}^{-1}$ in both experimental groups (C10 and C20). The presence of peak at this particular wavenumber in the control group ascertains the presence of unsaturated unpolymerized carbon-carbon double bond $(C=C)$. Apart from the formation of a three-dimensional network, the degree of conversion (DC) of the comonomers into copolymers is important in dentistry. The DC has influence on

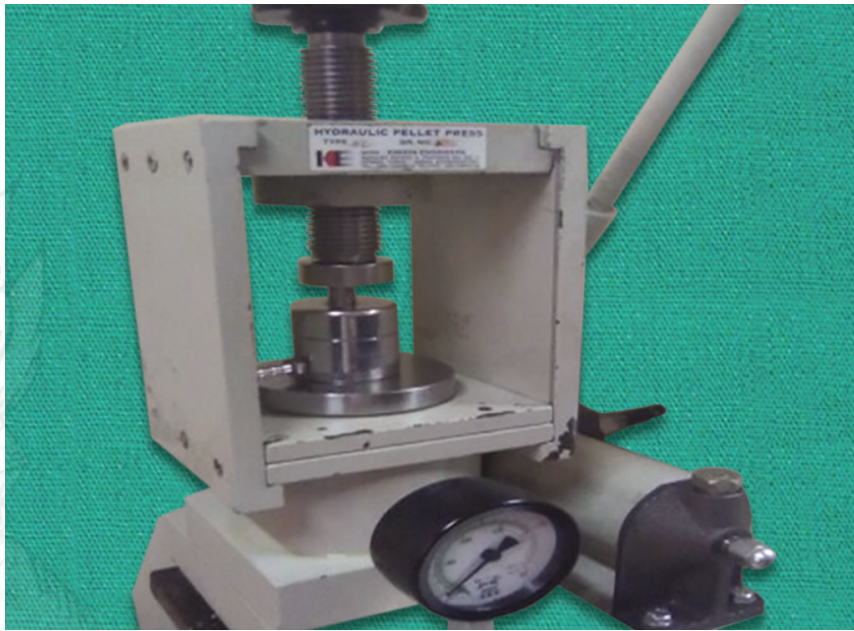

Fig. 3: Pellet forming hydraulic press

the material's properties and biocompatibility. Lower the DC, higher the amount of uncured $(C=C)$ monomers and additives. ${ }^{21}$ The presence of uncured $\mathrm{C}=\mathrm{C}$ double bond in the control group CO may be ascribed to the fact that the polymerization never

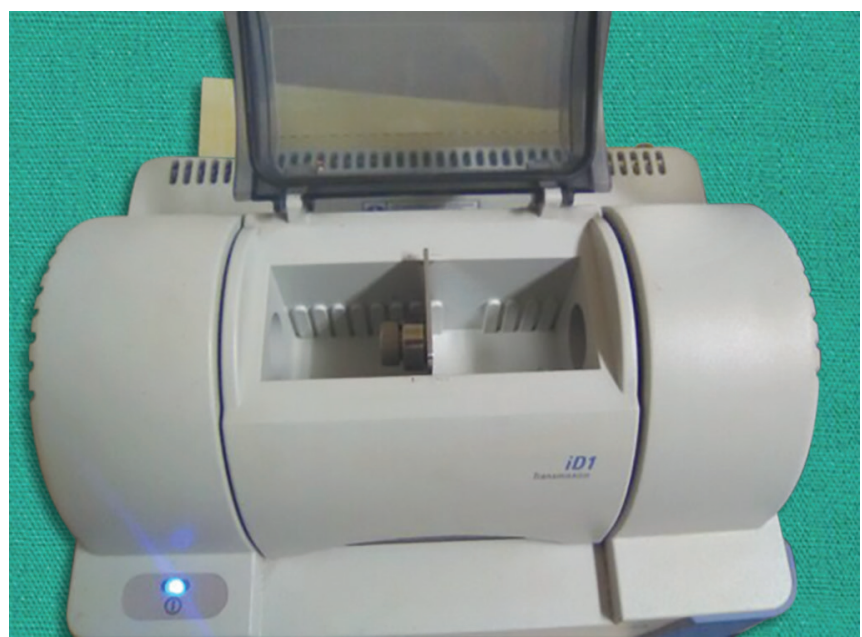

Fig. 4: Pellet holder inside FTIR spectrometer 


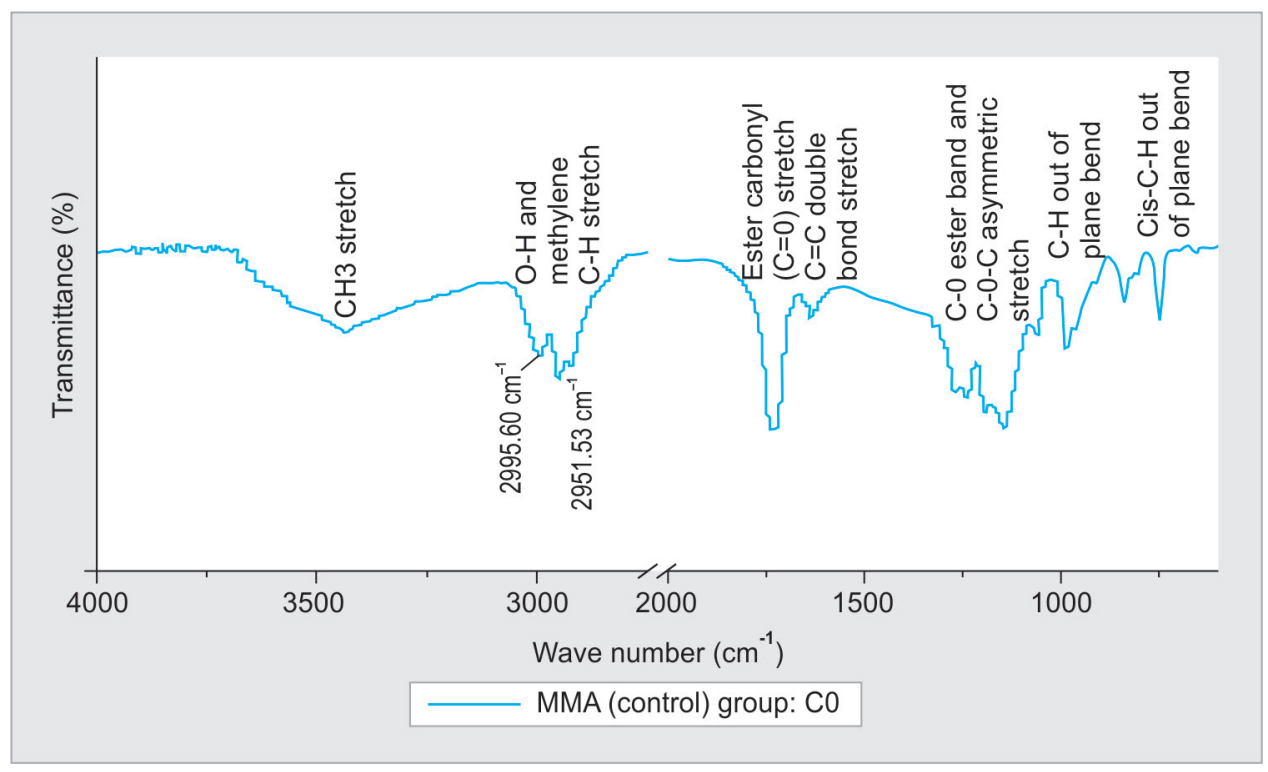

Fig. 5: FTIR spectrum of group: C0 (control)

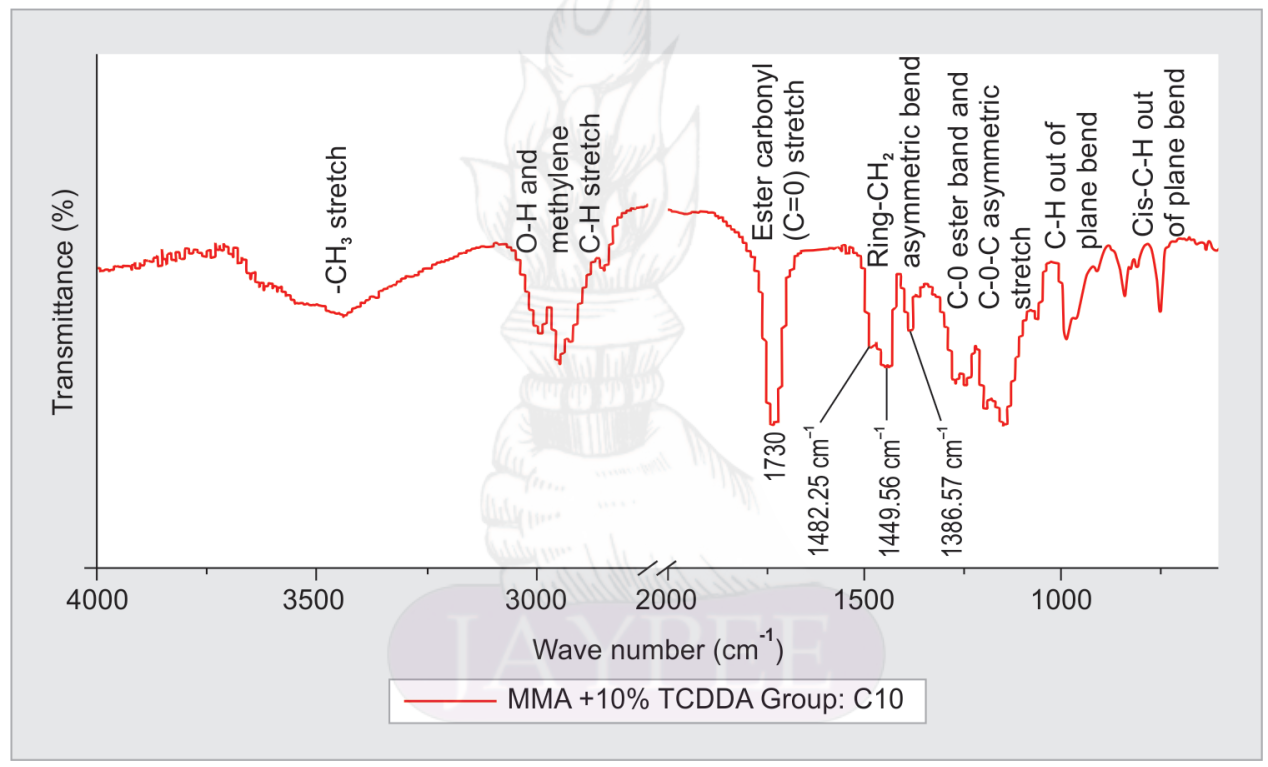

Fig. 6: FTIR spectrum of group: C10

reaches $100 \%$ DC. However, the final DC in specimens depends on the chemical composition of monomer, curing temperature, curing time, and operating room atmosphere. ${ }^{17}$ The $D C$ of $C=C$ double bond generally falls in the range of $55-65 \% .{ }^{22}$ However, this does not mean that $35-45 \%$ of the monomers are uncured. On the contrary, it means that $35-45 \%$ of the $C=C$ double bonds are uncured. It is possible that TCDDMDA bearing two $C=C$ double bonds may react with one of their $C=C$ double bonds with the other remaining unreacted.

Though the DC is never being complete, the absence of peak at $1637.34 \mathrm{~cm}^{-1}$ in both experimental groups may be ascribed to near-total conversion of monomers into polymers. The DC in diacrylates may be improved by increasing the distance between the reactive terminal groups and increasing molecular weight. ${ }^{18}$ The tricyclodecane three-ring central group offers steric hindrance effect that slows the rate of polymerization and facilitates the monomeric conversion to polymer thereby reducing the residual unpolymerized monomer $(\mathrm{C}=\mathrm{C}$ double bond) content in the final polymerized specimens. ${ }^{16}$ Al-Ali and Kassab-Bashi ${ }^{18}$ studied the FTIR spectroscopy of new copolymers of acrylic denture base materials by substituting methacrylic acid, butyl methacrylate, and ethyl acrylate in MMA at different concentrations. Addition of these monomers in MMA led to the disappearance of the $\mathrm{C}=\mathrm{C}$ bond, and, thus, corroborating the result of present study.

The second difference is the appearance of two new moderate peaks at $1482.25 \mathrm{~cm}^{-1}$ and $1449.56 \mathrm{~cm}^{-1}$ in both experimental groups. This is attributed to the ring $-\mathrm{CH}_{2}$ asymmetric bending ( $\mathrm{C}-\mathrm{H}$ deformation) vibrations. TCDDMDA contains a rigid, thermally stable cycloaliphatic molecule bearing bridged, fused-rings (tricyclic rings) with a three-dimensional structure that contributes 


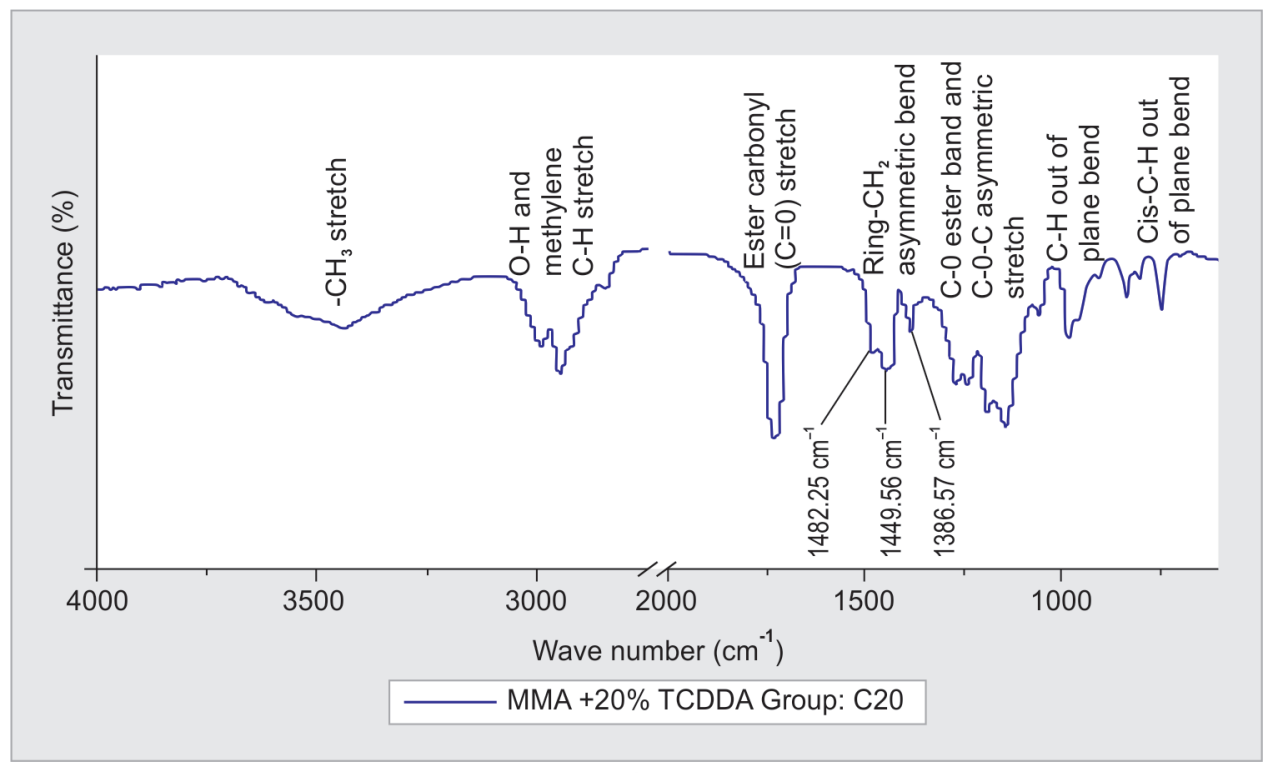

Fig. 7: FTIR spectrum of group: C20

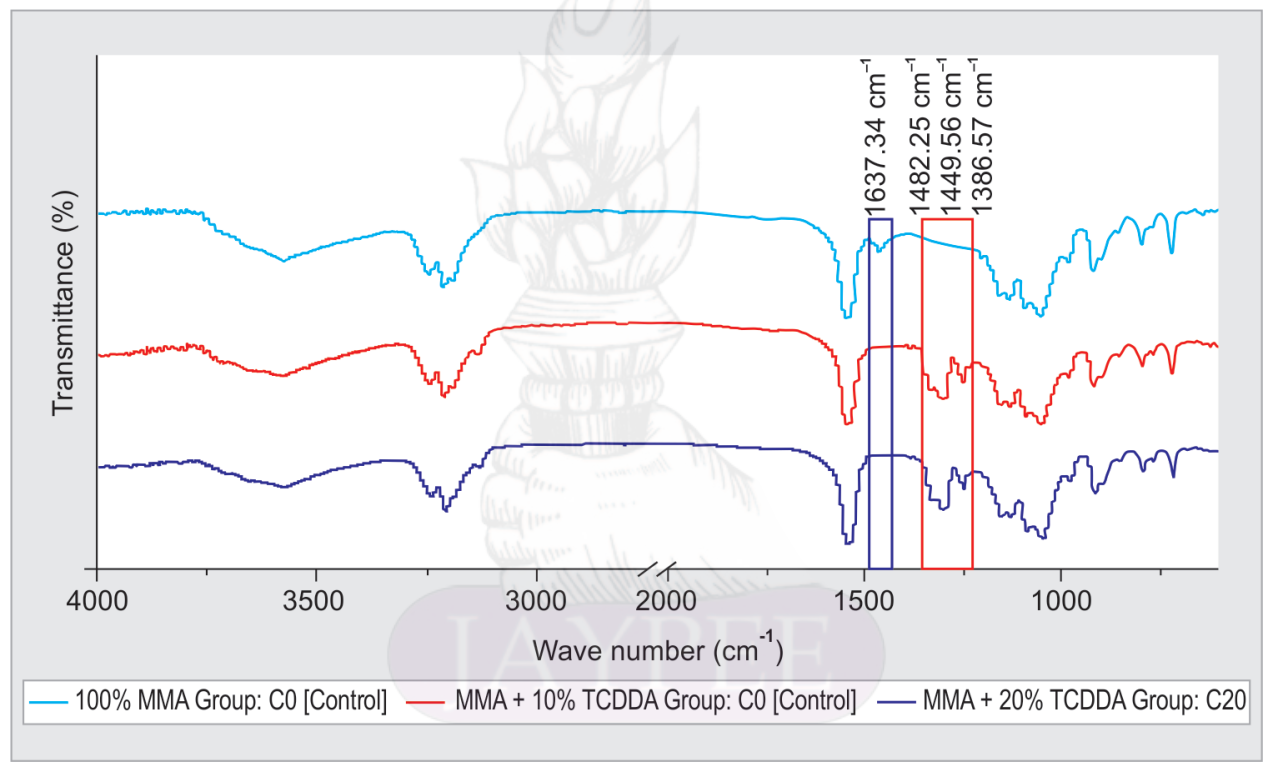

Fig. 8: Spectral differences between groups

to high glass-transition temperature $\left(T_{g}\right)$ values and improved properties. ${ }^{23}$ Hence, from this present study, it is ascertained that the cycloaliphatic-fused rings of TCDDMDA is responsible for the appearance of peculiar peaks in the FTIR spectra of experimental groups. Nonappearance of these characteristic peaks in the control group $\mathrm{CO}$ indicates the absence of cycloaliphatic ring structure in MMA. There is no significant difference between the transmitted spectra of both experimental groups.

The appearance of another new peak at $1386.57 \mathrm{~cm}^{-1}$ confirms the formation of a new structure of copolymer. A copolymer is two or more chemically different monomers, each with some desirable property combined to yield specific physical properties of a polymer. The two chemically different monomers used were MMA and TCDDMDA. In a copolymer, the relative number and the position of the different types of repeating units may vary among the individual macromolecules. ${ }^{24}$ The cured polymer, in most instances, contains a mixture of homopolymers and copolymers, with varying degrees of polymerization or copolymerization. Copolymerization also inhibits the aligning ability of polymeric chains to each other. Copolymers can vary in the molecular sequence and arrangement among the repeating random block and graft units. Copolymerization can have a very strong influence on the physical and mechanical properties of the resulting resin, changing them considerably from those of the respective homopolymers.

MMA, acrylic esters, and methacrylic esters all copolymerize readily, with little inhibition between monomer pairs. Grafting of various polymer segments onto a linear chain provides an important mechanism for modifying or tailor-making macromolecules to obtain required properties for specific uses. Block and graft copolymers often show improved impact strength. 
Table 1: Chemical characterizations of the groups

\begin{tabular}{llll}
\hline & & \multicolumn{2}{c}{ Groups } \\
\cline { 3 - 4 } Wave number $\left(\mathrm{cm}^{-1}\right)$ & Functional group & Control Experimental \\
\cline { 3 - 4 } $3436.38\left(-\mathrm{CH}_{3}\right)$ & Methyl stretch & $\mathrm{C} 10$ & $\mathrm{C} 20$ \\
$2995.60(\mathrm{O}-\mathrm{H})$ & Alcohol & Present Present Present \\
$2951.53(\mathrm{C}-\mathrm{H})$ & Methylene stretch & Present Present Present \\
$1731.79(\mathrm{C}=\mathrm{O})$ & Carbonyl stretch & Present Present Present \\
$1637.34(\mathrm{C}=\mathrm{C})$ & Alkenyl stretch & Present Present Present \\
$1500-1400\left(\right.$ ring $\left.-\mathrm{CH}_{2}\right)$ & Cyclo-alkane aliphatic ring asymmetric bend & Absent Present Present \\
1386.57 & Copolymer & Absent Present Present \\
$1271.44(\mathrm{C}-\mathrm{O})$ & Ester band & Present Present Present \\
$1147.82(\mathrm{C}-\mathrm{O}-\mathrm{C})$ & Ether asymmetric stretch & Present Present Present \\
$989.74,963.76,841.50(\mathrm{C}-\mathrm{H})$ & Aromatic; out of plane bend & Present Present Present \\
$750.92(\mathrm{cis}-\mathrm{C}-\mathrm{H})$ & Methylene; out of plane bend & Present Present Present \\
\hline
\end{tabular}

Therefore, MMA containing TCDDMDA might possess higher impact strength than the conventional MMA. In small quantities, these copolymers also can modify the adhesive properties of resins, as well as their surface characteristics. ${ }^{24}$ Another plausible explanation for copolymerization between MMA and TCDDMDA could be the ability of the TCDDMDA monomer to incorporate new functional groups due to its dual reactivity and to graft new polymeric or copolymeric chains.

Rodriguez et al. ${ }^{19}$ demonstrated the copolymerization of tertbutyl aminoethyl methacrylate (TBAEMA) with HC-PMMA. The evidence of this fact was the presence of new absorbance peaks in the FTIR spectral region $1300-1325 \mathrm{~cm}^{-1}$ of specimens containing TBAEMA (1\%, 2\%, 3\%, and 4\%), which were absent in the control group. Al-Ali and Alammari ${ }^{18}$ confirmed the copolymerization by substituting methacrylic acid, butyl methacrylate, and ethyl acrylate individually in MMA at 5\%, 10\%, and 15\% concentrations, respectively. A new transmittance peak appeared at $2358 \mathrm{~cm}^{-1}$ which confirmed the formation of new structure of copolymer, and this peak did not appear in the control group. Spasojevic et al. ${ }^{20}$ ascertained copolymerization by substituting di-methyl itaconate and di- $n$-butyl itaconate individually in MMA at $2.5 \%$ and $10 \%$ concentrations, respectively. A new transmittance peak at $1266 \mathrm{~cm}^{-1}$ (C-O-C asymmetric stretching vibration) confirmed the presence of copolymeric units while this peak was absent in the control group. Hence, from the above literature, it is inferred that MMA is one such monomer which can copolymerize with multitude monomers.

Cross-linkage provides a sufficient number of bridges between linear macromolecules to form a three-dimensional network that decreases water sorption and solubility and increases the strength and rigidity of the polymer. Characteristic features of polycycloaliphatic compounds include water or moisture tolerance, chemical resistance, poor wettability, impact resistance, increased surface hardness, and cross linkage. Polycycloaliphatic compounds, especially tricyclodecane dimethanol, are used to synthesize crosslinkers in the industrial field. ${ }^{23}$

This cross-linking capacity of cycloaliphatic compound has been exploited in the field of restorative dentistry as the filling material which offsets the polymerization shrinkage and improves the physico-mechanical properties. ${ }^{21,25-29}$ However, the effects of these compounds have not been studied or encountered in the field of prosthodontics yet. Hence, in this present study, TCDDMDA, a tricycloaliphatic compound, was selected to study the chemical characterization of HC-PMMA when incorporated into MMA.

In this present study, substituting concentrations of TCDDMDA in MMA selected were $10 \%$ and $20 \%$. In majority of in vitro studies regarding the monomer modification, the substituting concentrations of experimental monomer in proprietary monomer were $10 \%$ and $20 \%$ with an average range from $2.5 \%$ to $50 \% \mathrm{v} / \mathrm{v}$. Since the copolymerization between TCDDMDA and MMA has been established, it paves way for future research regarding the effect of this modified monomer on physico-mechanical properties and biocompatibility of HC-PMMA.

The present research is a triple-blinded study. The investigator, specimens, and operator were blinded by concealing the concentration of TCDDMDA substituted in MMA to avoid expectation bias that might breach into the result. A sample size of 10 specimens per group was arrived from the previous researches on FTIR or chemical characterization. ${ }^{17,18}$ Numerous testing methodologies and parameters are available for the analysis of polymers. FTIR is the most commonly used method for characterizing the chemical structure, assessing the presence of $\mathrm{C}=\mathrm{C}$ bonds, and characterizing copolymers and surface compositions. ${ }^{30}$

The modern FTIR spectrometer has three vital advantages over a typical dispersive infrared spectrometer. The multiplex advantage means multiple scans can be completed and averaged on an FTIR in a shorter time than one scan on most dispersive instruments. In this study, 10 scans were completed and averaged. Throughput advantage means more light energy reaches the specimen. The instrument does not limit the amount of light reaching the detector using a slit. FTIR spectrometer uses laser as a reference signal to control velocity of the moving mirror and to time the collection of data points throughout the mirror stroke. A constant sine-wave of laser provides the reference for both precision and accuracy of the infrared spectrometer. This is called as precision advantage. These advantages of FTIR make it as a standard tool and having largely replaced dispersive instruments by the mid-1980s. Therefore, in the current study, FTIR was used for chemical characterization of HC-PMMA with MMA monomer modified with TCDDMDA.

In the present study, concentrations of $10 \%$ and $20 \%$ of TCDDMDA were only substituted with MMA. Copolymerizing capability of TCDDMDA more than $20 \%$ was not experimented because it might affect the properties of the material. Further studies are to be carried out by increasing the concentration of 
TCDDMDA with MMA and its effect on the physico-mechanical properties and biocompatibility. Differences in the dough forming time between the groups were not considered.

\section{Conclusion}

Within the limitations of this study, the following conclusions were deduced:

- TCDDMDA copolymerizes with MMA when substituted at concentrations of $10 \%$ and $20 \%$.

- Maximum of $20 \%$ concentration of TCDDMDA can be substituted with MMA.

- Addition of TCDDMDA in MMA decreased the uncured residual monomer.

- TCDDMDA may be used as cross-linking agent in the monomers of denture base resins to improve the physico-mechanical properties.

\section{Clinical Significance}

Copolymerization of TCDDMDA with MMA would lead to the development of new monomeric composition for the fabrication of dentures possessing better mechanical properties and biocompatibility.

\section{References}

1. Ranganath $L M$, Shet RGK, et al. The effect of fiber reinforcement on the dimensional changes of polymethyl methacrylate resin after processing and after immersion in water: An in-vitro study. J Contemp Dent Pract 2011 Jul-Aug;12(4):305-317. DOI: 10.5005/ jp-journals-10024-1051.

2. Anderson GC, Schulte JK, et al. Dimensional stability of injection and conventional processing of denture base acrylic resin. J Prosthet Dent 1988 Sept;60(3):394-398. DOI: 10.1016/0022-3913(88)90292-2.

3. Anusavice KJ. Phillips' science of dental materials, 10th ed., USA: WB Saunders Company; 1996. pp. 237-272.

4. Becker $C M, S$ mith $D E$, et al. The comparison of denture base processing techniques. Part II. Dimensional change due to processing. J Prosthet Dent 1977 Apr;37(4):450-459. DOI: 10.1016/0022-3913(77)90147-0.

5. Craig RG, O'Brien WJ, et al. Dental-materials properties and manipulation, 4th ed., St Louis, USA: CV Mosby Co; 1990. pp. 272-296.

6. Dabreo EL, Herman P. A new method of measuring dimensional change. J Prosthet Dent 1991 May;65(5):718-722. DOI: 10.1016/00223913(91)90212-F.

7. Degee AJ, Tenharkel EC, et al. Measuring procedure for the determination of the three-dimensional shape of dentures.J J Prosthet Dent 1979 Aug;42(2):149-153. DOI: 10.1016/0022-3913(79)90164-1.

8. Dixon DL, Breeding LC, et al. Linear dimensional variability of three denture base resins after processing and in water storage. J Prosthet Dent 1992 July;68(1):196-200. DOI: 10.1016/0022-3913(92)90304-S.

9. Garfunkel E. Evaluation of dimensional change in complete denture processed by injection-pressing and the pack-and-press technique. J Prosthet Dent 1983 Dec;50(6):757-761. DOI: 10.1016/00223913(83)90085-9.

10. Hardy F. Comparison of fluid resin and compression molding methods in processing dimensional change. J Prosthet Dent 1978 Apr;39(4):375-377. DOI: 10.1016/S0022-3913(78)80150-4.
11. Firtell DN, Green AJ, et al. Posterior peripheral seal distortional related to processing temperature. J Prosthet Dent 1981 June;45(6):598-601. DOI: 10.1016/0022-3913(81)90418-2.

12. Jagger $D C$, Harrison $A$, et al. The reinforcement of dentures. J Oral Rehab 1996;26:185-194. DOI: 10.1046/j.1365-2842.1999.00375.x.

13. Rodford R. The development of high impact strength denture-base materials. J Dent 1986;14:214-217. DOI: 10.1016/0300-5712(86)90004-7.

14. Vallittu PK. A review of fiber-reinforced denture base resins. J Prosthodont 1996 Dec;5:270-276. DOI: 10.1111/j.1532-849X.1996.tb00511.x.

15. Ajay R, Suma K, et al. Monomer modifications of denture base acrylic resin: A systematic review and meta-analysis. J Pharm Bioall Sci 2019 May;11:S112-S125. DOI: 10.4103/JPBS.JPBS_34_19.

16. Anusavice KJ, Shen C, et al. Phillips' science of dental materials, 12th ed., St. Louis, Missouri: Saunders Elsevier; 2013. pp. 522.

17. Mahfooz AM, Alammari MR. The use of Fournier Transform Infrared (FTIR) spectroscopic analysis and cell viability assay to assess pre-polymerizes CAD/CAM acrylic resin denture base materials. Int J Pharm Res Allied Sci 2018;7(2):111-118.

18. Al-Ali AAS, Kassab-Bashi TY. Fournier transform infrared (FTIR) spectroscopy of new copolymers of acrylic denture base materials. Int J Enhanced Res Sci Tech Eng 2015 Apr;4(4):172-180.

19. Rodriguez $L S$, Paleari $A G$, et al. Chemical characterization and flexural strength of a denture base acrylic resin with monomer 2-tertbutylaminoethyl methacrylate. J Prosthodont 2013;22:292-297. DOI: 10.1111/j.1532-849X.2012.00942.x.

20. Spasojevic $\mathrm{P}$, Zrilic M, et al. The mechanical properties of a poly(methyl methacrylate) denture base material modified with dimethyl itaconate and di-n-butyl itaconate. Int J Polymer Sci 2015;June;2015: 1-9. DOI: $10.1155 / 2015 / 561012$.

21. Durner J, Obermaier J, et al. Correlation of the degree of conversion with the amount of elutable substances in nano-hybrid dental composites. Dent Mater 2012;28:1146-1153. DOI: 10.1016/ j.dental.2012.08.006.

22. Miletic VJ, Santini A. Remaining unreacted methacrylate groups in resin-based composite with respect to sample preparation and storing conditions using micro-Raman spectroscopy. J Biomed Mater Res Part B: ApplBiomater 2008;87B:468-474. DOI: 10.1002/jbm.b.31128.

23. Deepak VD, Rajan J, et al. Hydrogen bonding and rate enhancement in the photoinduced polymerization of telechelic urethane methacrylates based on a cycloaliphatic system: tricyclodecane dimethanol. J PolymSci Part A: PolymChem 2006;44:4384-4395.

24. Anusavice KJ. Phillips' science of dental materials, 11th ed., St. Louis, Missouri: Saunders Elsevier; 2003. pp. 162-164.

25. Endo T, Finger WJ, et al. Surface texture and roughness of polished nanofill and nanohybrid resin composites. Dent Mater J 2010;29(2):213-223. DOI: 10.4012/dmj.2009-019.

26. Frauscher KE, llie N. Depth of cure and mechanical properties of nanohybrid resin-based composites with novel and conventional matrix formulation. Clin Oral Invest 2012 Dec;16:1425-1434. DOI: 10.1007/ s00784-011-0647-3.

27. Ilie N, Hickel R. Resin composite restorative materials. Aust Dent J 2011;56(1 Suppl):59-66. DOI: 10.1111/j.1834-7819.2010.01296.x.

28. Marchesi G, Breschi L, et al. Contraction stress of low-shrinkage composite materials assessed with different testing systems. Dent Mater 2010;26:947-953. DOI: 10.1016/j.dental.2010.05.007.

29. Schimdt C, llie N. The mechanical stability of nano-hybrid composites with new methacrylate monomers for matrix compositions. Dent Mater 2012;28:152-159. DOI: 10.1016/j.dental.2011.11.007.

30. International Organization for Standardization, ISO 10993-18. Biological Evaluation of medical devices - Part 18: Chemical characterization of materials. British Standards: ISO; 2009. 